\title{
Band Alignments in $\operatorname{In}_{\mathrm{x}} \mathrm{Ga}_{1-\mathrm{x}} \mathrm{P} / \mathrm{GaAs}$ Heterostructures Investigated by Pressure Experiments
}

\author{
J. Martínez-Pastor (a, c), J. Camacho (a), C. Rudamas (a), A. Cantarero (a), \\ L. GonZÁlez (b), and K. SyASSEN (c)
}

(a) Instituto de Ciencia de los Materiales, Universidad de Valencia, Apdo. Correas 2085, E-46071 Valencia, Spain

Tel.: +34-963864793; Fax +34-963983633; e-mail: martinep@uv.es

(b) Instituto de Microelectrónica de Madrid, CNM-CSIC, PTM, E-28760 Tres Cantos (Madrid), Spain

Tel.: +34-918060700; Fax +34-918060701; e-mail: luisa@imm.cnm.csic.es

(c) Max-Planck-Institut für Festkörperforschung, Heisenbergstr. 1, D-70569 Stuttgart, Germany

Tel.: +49-711-6891446, Fax: +49-711-6891444; e-mail: syassen@servix.mpi-stuttgart.mpg.de

$\operatorname{In}_{x} \mathrm{Ga}_{1-x} \mathrm{P} / \mathrm{GaAs}(x=0.541$ and 0.427$)$ heterostructures, grown by Atomic Layer Molecular Beam Epitaxy (ALMBE) on low temperature substrates, have been characterised by pressure-dependent and time-resolved photoluminescence experiments. The excitonic optical transitions and recombination dynamics are both influenced by the particular band alignments of these systems. The valence band offset has been found to have approximately the same absolute value $\left(\Delta E_{\mathrm{VB}} \approx 380 \mathrm{meV}\right)$, independent of the In content of the alloy in the barrier, whereas the conduction band offset varies appreciably depending on the alloy band gap. The huge valence band offset implies a strong asymmetry in the confinement of carriers, affecting the exciton recombination dynamics in the quantum wells.

Introduction A great deal of work has been devoted in the last years to the growth, control and characterisation of InGaP epitaxial layers lattice-matched to GaAs. InGaP is a substitute for AlGaAs which usually contains a larger concentration of deep traps and has a higher reactivity with oxygen. As a consequence, InGaP/GaAs heterostructures are now the basis for electronic and optoelectronic devices, i.e. heterojunction transistors [1], laser diodes [2] or tandem solar cells [3]. Another peculiarity of the InGaP system is that the In-Ga compositional ratio can be tuned around the latticematched composition. In this way, different strain conditions can be realised in InGaP layers [4]. In addition, major changes in the optical properties can be induced [5, 6] if pronounced ordering and phase separation effects, exhibiting a periodic arrangement in the crystal, could be controlled during crystal growth.

From the point of view of basic research, the InGaP/GaAs heterojunctions offer important differences with respect to the $\mathrm{AlGaAs} / \mathrm{GaAs}$ ones. The main difference arises from the band alignment between InGaP and GaAs. The valence band offset (VBO) in the In $\mathrm{GaP} / \mathrm{GaAs}$ system is still a controversial subject, and different values have been obtained from different experiments (see for example Refs. [5, 7] and literature cited therein). Optical experiments on MBE grown samples indicate a huge VBO, leading to 
a strong asymmetry in the confinement of holes and electrons [8]. Nevertheless, the values for the band offsets are not well established and appreciable differences appear even for values derived from high-pressure photoluminescence (PL) experiments [9, 10]. It is clear that optical experiments need a model in order to account for the optical transitions. However, this is not the main origin of the discrepancies. More important are the differences in the structural quality of the alloy obtained by different growth methods or conditions. Compositional and ordering effects in the alloy can lead to important localisation effects of excitons because of the different band alignment between the CuPt-like ordered InGaP alloy and GaAs (type-II band alignment) [5, 11] with respect to the case of the disordered alloy (type-I).

The aim of this work is to investigate the VBO between InGaP and GaAs using high-pressure PL experiments. The InGaP alloy in our samples is basically free of ordered domains such that the quantum well (QW) emission energies are determined by the average composition [8]. A tight binding model has been used to calculate optical transitions, below and above the $\Gamma-\mathrm{X}$ crossover point, based on known band structure parameters of bulk GaAs, InP and $\mathrm{GaP}$ and as a function of the $\mathrm{VBO}$ between the alloy and GaAs. This kind of model is useful to validate the strategy followed in this paper to find the band alignment energies. The work has been performed for InGaP alloys with two different In:Ga ratios (42.7:57.3 and 54.1:45.9) around the latticematched composition (48:52), leading to biaxial tensile or compressive strain in the layers. Using two different alloy compositions allows us to demonstrate that the band alignment is also dependent on composition, as has been proposed in previous works $[4,8]$. The band offset characteristics for InGaP lattice-matched to GaAs should be intermediate between the results obtained for the two compositions studied here.

Samples and Experiment Two $\operatorname{In}_{x} \mathrm{Ga}_{1-x} \mathrm{P}$-based heterostructures $(x=0.541$ and 0.427 ) have been grown by ALMBE at a substrate temperature of $420{ }^{\circ} \mathrm{C}$. Each sample contains seven quantum wells (QWs) of 3, 5, 8, 12, 17, 25 and 50 monolayers $(1 \mathrm{ML}=0.283 \mathrm{~nm})$ thick, decoupled by thick enough InGaP barriers. PL spectra of both samples were measured at $10 \mathrm{~K}$ as a function of pressure $(0$ to $6 \mathrm{GPa})$ in a diamond anvil cell. The excitation source was the $514.5 \mathrm{~nm}$ line of an $\mathrm{Ar}^{+}$laser and several excitation densities were used in the range 20 to $2000 \mathrm{~W} / \mathrm{cm}^{2}$. Time-resolved PL measurements at $10 \mathrm{~K}$ have been performed by using above-barrier excitation by a mode-locked Nd:YAG pumped dye laser and signal recording by a synchroscan streak camera.

Results and Discussion Figure 1 shows some PL spectra of the $\operatorname{In}_{0.427} \mathrm{Ga}_{0.573} \mathrm{P} / \mathrm{GaAs}$ sample for different pressures, below and above the $\Gamma-\mathrm{X}$ crossover point. At low excitation power the emission from six of the seven QWs is well observed below the crossover (occurring between 2.5 and $2.9 \mathrm{GPa}$, depending on the QW width). The PL from the thickest QW (50 ML) is only observed for excitation densities above $100 \mathrm{~W} / \mathrm{cm}^{2}$. The overall optical quality of the different QWs is quite good judged by the narrow full width at half maximum of the PL lines, ranging between 4 and $13 \mathrm{meV}$ (increasing with decreasing well width). Above the crossover pressure, the PL intensity of the QWs drops by around two orders of magnitude with respect to values below that pressure. A similar behaviour is observed in the $\operatorname{In}_{0.541} \mathrm{Ga}_{0.459} \mathrm{P} / \mathrm{GaAs}$ sample, but the crossover pressure occurs around $3.5 \mathrm{GPa}$ due to the higher In content in the alloy layer. A near 


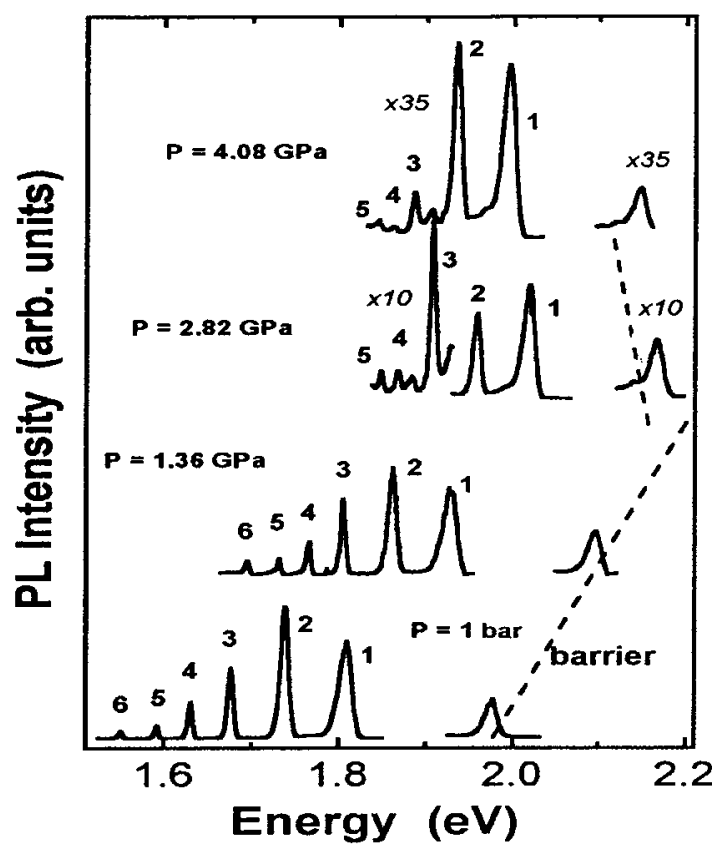

Fig. 1. PL spectra of the $\operatorname{In}_{0.427} \mathrm{Ga}_{0.573} \mathrm{P} /$ GaAs sample taken at different pressures

linear increase (decrease) of the optical transition energies is observed below (above) the crossover pressure.

In Fig. 2 we show the energy difference between the type-II $\left(E_{\mathrm{X}}^{\mathrm{QW}}\right)$ and type-I $\left(E_{\Gamma}^{\mathrm{QW}}\right)$ optical transitions in each QW, extrapolated to zero pressure. The experimental values have been deduced from the linear fits of the pressure dependence of the PL peak energies of the different QWs. These values were also corrected for the exciton binding energies and Stokes shifts. A noticeable difference exists in the QW thickness dependence of the two samples, which cannot be explained if the band offset ratios $\left(Q_{\mathrm{C}}\right.$ or $\left.Q_{\mathrm{V}}\right)$ were independent of the composition, as is the case in the AlGaAs/ GaAs system.

The energy difference between type-II and type-I transitions, extrapolated to zero pressure, is the most common and useful way to extract the VBO in QW structures. After having corrected for the exciton binding energy and localisation, only two parameters enter into that energy difference, the $\mathrm{VBO} \Delta E_{\mathrm{VB}}$ and the electron confinement energy $E_{\mathrm{e}}^{\mathrm{QW}}(L)$ in a $\mathrm{QW}$ with thickness $L$

$$
\left[E_{\mathrm{X}}^{\mathrm{QW}}-E_{\Gamma}^{\mathrm{QW}}\right](L)=\left[E_{\mathrm{X}}^{\mathrm{InGaP}}-E_{\Gamma}^{\mathrm{GaAs}}\right]-\Delta E_{\mathrm{VB}}-E_{\mathrm{e}}^{\mathrm{QW}}(L) .
$$

The electron subband energy should decrease with increasing $L$ from the top of the well ( $L=0$, barrier conduction band minimum) to zero when $L$ tends to infinity. Here, the GaAs conduction band is taken as the reference energy. An approximate expres-

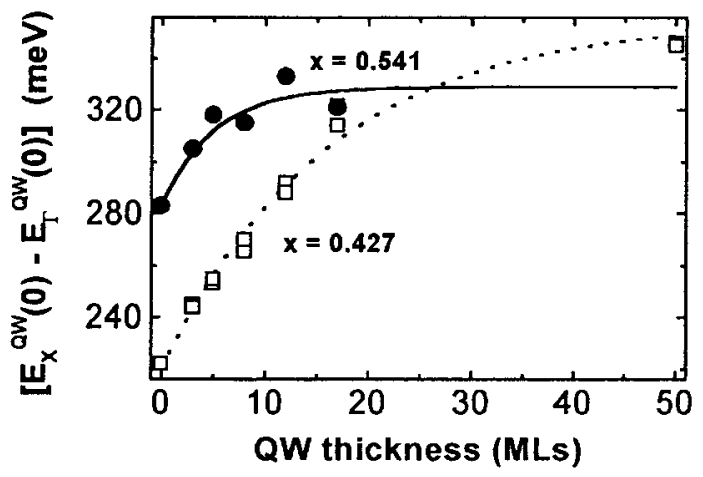

Fig. 2. QW thickness dependence of the energy difference between type-II and type-I optical transitions, extrapolated to zero pressure. The continuous and dotted lines represent the best fits to Eq. (2) 
sion accounting for this variation is

$$
\left[E_{\mathrm{X}}^{\mathrm{QW}}-E_{\Gamma}^{\mathrm{QW}}\right](L)=\left[E_{\mathrm{X}}^{\mathrm{InGaP}}-E_{\Gamma}^{\mathrm{InGaP}}\right]+\Delta E_{\mathrm{CB}}\left[1-\mathrm{e}^{L / L_{0}}\right],
$$

where $\Delta E_{\mathrm{CB}}$ is the conduction band offset $(\mathrm{CBO})$ and $L_{0}$ is a characteristic QW width enabling the saturation of Eq. (2). The fit of Eq. (2) to the experimental values obtained in pressure experiments allows us to deduce the conduction band gap offset ratio

$$
Q_{\mathrm{C}}=\frac{\Delta E_{\mathrm{CB}}}{\Delta E_{\mathrm{G}}}=\frac{\Delta E_{\mathrm{G}}-\Delta E_{\mathrm{VB}}}{\Delta E_{\mathrm{G}}},
$$

where $\Delta E_{\mathrm{G}}=\left[E_{\Gamma}^{\mathrm{InGaP}}-E_{\Gamma}^{\mathrm{GaAs}}\right]$. Figure 2 shows the fit of Eq. (2) to the experimental data (continuous and dotted lines for $x=0.541$ and 0.427 samples, respectively). The parameters obtained from the fit are: $\left[E_{\mathrm{X}}^{\mathrm{InGaP}}-E_{\Gamma}^{\mathrm{InGaP}}\right]=220(3) \mathrm{meV}, \Delta E_{\mathrm{CB}}=135$ (7) $\mathrm{meV}$ and $L_{0}=16(2) \mathrm{MLs}$ for the sample with an In content $x=0.427$, and $\left[E_{\mathrm{X}}^{\mathrm{InGaP}}-E_{\Gamma}^{\mathrm{InGaP}}\right]=$ 283 (2) meV, $\Delta E_{\mathrm{CB}}=46$ (6) meV and $L_{0}=5$ (1) MLs for the sample with $x=0.541$.

In order to test the validity of Eq. (2), we have performed a tight-binding calculation by introducing the same conduction band offset ratio as the one obtained from the fit. The results of the tight-binding calculations practically coincide with the results of the fit. This consistency proves the validity of using Eq. (2) to obtain a good value of $Q_{\mathrm{C}}$, when the pressure experiments are performed on a sample containing a sufficient number of QWs (decoupled) of different thicknesses.

From the results of the fitting and by means of Eq. (3) and the experimental $\Delta E_{\mathrm{G}}=$ $515 \mathrm{meV}(425 \mathrm{meV})$, we obtain a $\mathrm{CBO}$ ratio $Q_{\mathrm{C}} \approx 0.26\left(Q_{\mathrm{C}} \approx 0.11\right)$ for $x=0.427$ $(x=0.541)$. Different CBO ratios were also assumed in previous works $[4,8]$, in order to explain with a simple model the heavy and light hole exciton transitions in the samples used in the present work. On the other hand, the valence band alignment between GaAs and InGaP is conserved, $\Delta E_{\mathrm{VB}} \approx 380$ (20) meV, independent of the barrier composition. This means that the band gap variation with In content is fully absorbed in the conduction band alignment. Our value of the VBO is similar to that found by Chen et al. [9] and slightly larger than the one found by Leroux et al. [10], who reported a value of $330 \mathrm{meV}$. In the case of an InGaP alloy lattice-matched to GaAs, the conduction band offset would be around 0.19 , by assuming the same absolute VBO and $\Delta E_{\mathrm{G}}=470 \mathrm{meV}$.

The strong asymmetry in the confinement of electrons and holes and the variation of the conduction band offset with In content in the alloy affect the exciton recombination dynamics. Figure 3 summarises the variation of the PL decay time $(T=10 \mathrm{~K})$ with the QW width for both samples studied. For QWs wells thinner than 12 MLs the decay time increases with decreasing thickness, whereas it is practically constant for thicker QWs.

To give a quantitative account for the well width dependence of the radiative excitonic lifetime we can calculate the oscillator strength of an optically allowed dipole transition. This magnitude is proportional to the square of the dipole matrix element connecting Bloch states in the valence and conduction bands, to the overlap of the envelope wave functions (along the growth direction) for the conduction and valence subbands squared, and to the squared $2 \mathrm{D}$ hydrogen-like exciton wave function taken at $r=0$ ( $r$ is the relative distance between the electron and hole). The latter is proportional to $a_{0}^{-2}$, where $a_{0}$ is the effective $2 \mathrm{D}$ exciton Bohr radius, which depends on the quantum well thickness. Taking into account that the lifetime is inversely proportional 


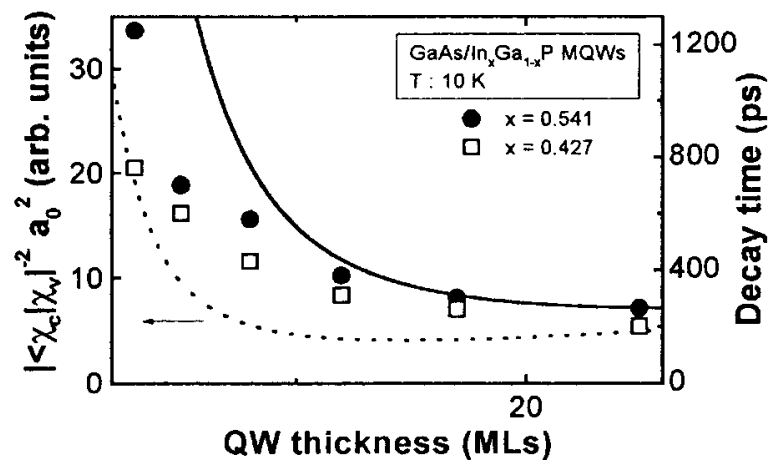

Fig. 3. PL decay time measured at $10 \mathrm{~K}$ as a function of the QW width in the two samples with different In content. Solid and dotted lines represent the calculated curves for both samples by using Eq. (4) and the same scale factor

to the oscillator strength of the transition and assuming the matrix element connecting the Bloch states to be independent of the well width, the PL decay time can be written as [13]

$$
\tau^{2 \mathrm{D}} \propto|\rangle \chi_{\mathrm{C}} \mid \chi_{\mathrm{V}}\left\langle\left.\right|^{-2} a_{0}^{2}\right.
$$

The calculations have been made for both $\operatorname{In}_{x} \mathrm{Ga}_{1-x} \mathrm{P} / \mathrm{GaAs}$ samples by using the CBO ratios deduced from the pressure experiments. The calculated curves (continuous and dotted lines in Fig. 3, for $x=0.541$ and 0.427 , respectively), multiplied by a scale factor which accounts for the dipole matrix element of Bloch states, reproduce approximately the experimental behaviour of the PL decay time measured in lower and higher In content samples as a function of the QW width. The dominant factor in Eq. (4), for relatively thin QWs, is the electron and hole wavefunction overlap integral, which is determined by the small $\mathrm{CBO}$ in the InGaP/GaAs system. The low electron barrier potential height produces shallow electron subbands, whereas the heavy hole states are deeper confined in the well in the QW thickness range examined here. As a consequence, the asymmetry between the wavefunction of the electrons and holes becomes more important when reducing the QW width. The 3D limit is rapidly reached even for relatively thick QWs, as compared to the case of the AlGaAs/GaAs system. The experimental PL decay times are smaller in the case of the sample with $x=0.427$, near a factor two for the narrowest QW. Such a difference should be correlated with the higher $\mathrm{CBO}$ in that sample.

Conclusions In this paper, the band gap alignment in the $\operatorname{In}_{x} \mathrm{Ga}_{1-x} \mathrm{P} / \mathrm{GaAs}$ system has been studied for two different In compositions. An operative model to obtain the VBO in this kind of heterostructures is presented (Eqs. (1) and (2)). The model is based on the QW width dependence of the energy difference between type-II and type-I optical transitions, extrapolated to zero. With this model, we have deduced an absolute valence band offset of $\Delta E_{\mathrm{VB}} \approx 380(20) \mathrm{meV}$, which is independent of the In content in the barrier alloy. On the other hand, the CBO varies appreciably with the alloy composition following the variation of the alloy band gap. The huge valence band offset implies a strong asymmetry in the confinement of carriers, which affects the exciton recombination in the quantum well when reducing the QW width. At the same time, the different CBO for different alloy compositions introduces important changes in the measured PL decay times, especially for narrow QWs. 
Acknowledgements J. M.-P. wishes to thank the European Union for a grant to use LENS facilities at Florence, and also Dr. Colocci and Dr. Vinattieri for their help. This work has been supported by the projects TIC96-1020-C02 and HA1997-0129 of the Ministry of Education of Spain.

\section{References}

[1] C. Jelen, S. Slivken, X.G. He, M. Razeghi, and S. Shastry, J. Vac. Sci. Technol. B 12, 1113 (1994).

[2] M. Jalonen, M. Toivonen, P. Savolainen, J. Köngäs, and M. Pessa, Appl. Phys. Lett. 71, 479 (1997).

[3] K.A. Bertness, S.R. Kurtz, D.J. Friedman, A.E. Kibbler, C. Kramer, and J.M. Olson, Appl. Phys. Lett. 65, 989 (1994).

[4] J. Martínez-Pastor, L. González, and Ph. Roussignol, Appl. Phys. Lett. 68, 2111 (1996).

[5] S. Froyen, A. Zunger, and A. Mascarenhas, Appl. Phys. Lett. 68, 2852 (1996).

[6] J. Zeman, G. Martinez, P.Y. Yu, and K. Uchida, Phys. Rev. B 55, R13428 (1997).

[7] M.S. Faleh, J. Tasselli, J.P. Bailbe, and A. Marty, Appl. Phys. Lett. 69, 1288 (1996).

[8] J. Martínez-Pastor, L. González, G. Aragón, Ch. Guenaud, and E. Deleporte, J. Appl. Phys. 84, 6832 (1998).

[9] J. Chen, J.R. Sites, I.L. Spain, M.J. Hafich, and G.Y. Robinson, Appl. Phys. Lett. 58, 744 (1991).

[10] M. Leroux, M.L. Fille, B. Gil, J.P. Landesman, and J.C. García, Phys. Rev. B 47, 6465 (1993).

[11] J. Zeman, G. Martínez, P.Y. Yu, and K. Uchida, Phys. Rev. B 55, R13428 (1997).

[12] J. MartíneZ-Pastor et al., to be published.

[13] U. Cebulla, G. Bacher, A. Forchel, G. Mayer, and W. T. Tsang, Phys. Rev. B 39, 6257 (1989). 